\title{
LA CONSTITUCION CANADIENSE DE 1980
}

Es bien sabido que las leyes fundamentales referidas al territorio y los ciudadanos canadienses están contenidas, hasta ahora, en el British Nortb American Act, acordado por el Parlamento británico el 1 de julio de 1867.

El Gobierno federal canadiense ha remitido al Parlamento una resolución para la repatriación de la Constitución al propio Canadá. Esta resolución fue discutida y rechazada recientemente en la conferencia que reunió en Ottawa a los primeros ministros de las diez provincias canadienses, presididos por el primer ministro federal Pierre Elliot Trudeau.

Tras este rechazo al proyecto constitucional de los jefes de Gobierno de las provincias, el Gobierno federal dispuso la convocatoria urgente del Parlamento para considerar la cuestión.

Además del tema de la repatriación, la resolución contiene cláusulas constitucionales modificadoras tan importantes como la Carta Canadiense de Derechos y Libertades y otras referentes todas ellas a problemas que son de una gran importancia en la política actual de Canadá: el tema de la redistribución de las riquezas entre provincias ricas y pobres, el problema de las minorías lingüísticas, etc.

Si la resolución propuesta es adoptada por el Parlamento, el Gobierno federal la someterá a la reina Isabel II de Inglaterra, solicitando del Parlamento británico que decrete las disposiciones contenidas en la citada resolución y transfiera a la autoridad canadiense todas las medidas contenidas en las leyes constitucionales británicas que se refieren a Canadá.

Ofrezco a continuación el texto íntegro del Proyecto de Resolución ${ }^{1}$, que en el momento de escribir esta pequeña introducción se encuentra en fase de segunda lectura en el Parlamento canadiense.

Fernando Ollero Butler

${ }^{1}$ Fuente: Embajada del Canadá. 
Projet de résolution portant adresse commune à Sa Majesté la Reine concernant la Constitution du Canada

Le Sénat et la Chambre des communes du Canada réunis en Parlement, considérant:

que le Parlement du Royaume-Uni a modifié à plusieurs reprises la Constitution du Canada à la demande et avec le consentement de celui-ci;

que, de par le statut d'État indépendant du Canada, il est légitime que les Canadiens aient tout pouvoir pour modifier leur Constitution au Canada;

qu'il est souhaitable d'inscrire dans la Constitution du Canada la reconnaissance de certains droits et libertés fondamentaux et d'y apporter d'autres modifications,

ont résolu de présenter respectueusement à Sa Majesté la Reine l'adresse dont la teneur suit.

A Sa Très Excellente Majesté la Reine, Très Gracieuse Souveraine:

Nous, membres du Sénat et de la Chambre des communes du Canada réunis en Parlement, fidèles sujets de Votre Majesté, demandons respectueusement à Votre Très Gracieuse Majesté de bien vouloir faire déposer devant le Parlement du Royaume-Uni un projet de loi ainsi conçu:

ANNEXE A

\section{LOI MODIFIANT LA CONSTITUTION DU CANADA}

Sa Très Excellente Majesté la Reine, considérant:

qu'à la demande et avec le consentement du Canada, le Parlement du Royaume-Uni est invité à adopter une loi visant à donner effet aux dispositions énoncées ci-après et que le Sénat et la Chambre des communes du Canada réunis en Parlement ont présenté une adresse demandant à Sa Très Gracieuse Majesté de bien vouloir faire déposer devant le Parlement du RoyaumeUni un projet de loi à cette fin,

sur l'avis et du consentement des Lords spirituels et temporels et des Communes réunis en Parlement, et par l'autorité de celui-ci, édicte:

Adoption de la

Loi constitu-

tionnelle des

1980

Cessation du pouvoir de légiférer pour le Canada

Version française

Titre abrégé
1. La Loi constitutionnelle de 1980, énoncée à l'annexe B, est édictée pour le Canada et y a force de loi. Elle entre en vigueur conformément à ses dispositions.

2. Les lois adoptées par le Parlement du Royaume-Uni après l'entrée en vigueur de la Loi constitutionnelle de 1980 ne font pas partie du droit positif du Canada.

3. La partie de la version française de la présente loi qui figure à l'annexe $\mathrm{A}$ a force de loi au Canada au même titre que la version anglaise correspondante.

4. Titre abrégé de la présente loi: Loi sur le Canada. 
ANNEXE B

LOI CONSTITUTIONNELLE DE 1980

PARTIE I

CHARTE CANADIENNE DES DROITS ET LIBERTES

\section{Garantie des droits et libertés}

1. La Charte canadienne des droits et libertés garantit les droits et libertés énoncés ci-après, sous les seules réserves normalement acceptées dans une société libre et démocratique de régime Droits et libertés au Canada parlementaire.

\section{Libertés fondamentales}

2. Chacun a les libertés fondamentales suivantes:

a) liberté de conscience et de religion;

Libertés

b) liberté de pensée, de croyance, d'opinion et d'expression, y compris la liberté de la presse et des autres grands moyens d'information;

c) liberté de réunion pacifique et d'association.

\section{Droits démocratiques}

3. Tout citoyen canadien a le droit de vote et est éligible aux élections législatives fédérales ou provinciales; ce droit ne peut, sans motif valable, faire l'objet d'aucune distinction ou restriction.

4. (1) Le mandat maximal de la Chambre des communes et Mandat des assemblées législatives est de cinq ans à compter de la date du rapport des brefs relatifs aux élections générales correspondantes.

(2) Le mandat de la Chambre des communes ou celui d'une assemblée législative peut être prolongé respectivement par le Parlement ou par la législature en question audelà de cinq ans en cas de guerre, d'invasion ou d'insurrection, réelles ou appréhendées, pourvu que cette prolongation ne fasse pas l'objet d'une opposition exprimée par les voix de plus du tiers des députés de la Chambre des communes ou de l'assemblée législative.

5. Le Parlement et les législatures tiennent une séance au Séance ann moins une fois tous les douze mois.

\section{Liberté de circulation et d'établissement}

6. (1) Tout citoyen canadien a le droit de demeurer au Ca- Droits des nada, d'y entrer ou d'en sortir. 
Droits généraux

Restriction

Vie, liberté et sécurité

Fouilles, perquisitions et saisies

Détention ou empresionnement

Arrestation ou đétention

Affaires criminelles et pénales
(2) Tout citoyen canadien et toute personne ayant le statut de résident permanent au Canada ont le droit:

a) de se déplacer dans tout le pays et d'établir leur résidence dans toute province;

b) de gagner leur vie dans toute province.

(3) Les droits mentionnés au paragraphe (2) sont subordonnés: a) aux lois et usages d'application générale en vigueur dans une province donnée, s'ils n'établissent entre les personnes aucune distinction fondée principalement sur la province de résidence antérieure ou actuelle;

b) aux lois prévoyant de justes conditions de résidence en vue de l'obtention des services sociaux publics.

\section{Garanties juridiques}

7. Chacun a droit à la vie, à la liberté et à la sécurité de sa personne; il ne peut être porté atteinte à ce droit qu'en conformité avec les principes de justice fondamentale.

8. Chacun a droit à la protection contre les fouilles, les perquisitions et les saisies abusives dont les motifs ne sont pas fondés sur la loi et qui ne sont pas effectuées dans les conditions que celle-ce prévoit.

9. Chacun a droit à la protection contre la détention ou l'emprisonnement dont les motifs ne sont pas fondés sur la loi et qui ne sont pas effectués dans les conditions que celle-ci prévoit.

10. Chacun a le droit, en cas d'arrestation ou de détention:

a) d'être informé dans les meilleurs délais des motifs de son arrestation ou de sa détention;

b) d'avoir recours sans délai à l'asistance d'un avocat;

c) de faire contrôler, par babeas corpus, la légalité de sa détention et d'obtenir, le cas échéant, sa libération.

11. Tout inculpé a le droit:

a) d'être informé dans les meilleurs délais de l'infraction précise qu'on lui reproche;

b) d'être jugé dans un délai raisonnable;

c) d'être présumé innocent tant qu'il n'est pas déclaré coupable, conformément à la loi, par un tribunal indépendant et impartial à l'issue d'un procès public et équitable;

d) de ne pas être privé d'une mise en liberté assortie d'un cautionnement raisonnable, sauf pour des motifs fondés sur la loi et dans les conditions que celle-ci prévoit;

e) de ne pas être déclaré coupable en raison d'une action ou d'une omission qui, au moment où elle est survenue, ne constituait pas une infraction;

f) de n'être poursuivi ou puni qu'une fois pour une infraction dont il a dejà été définitivement acquitté ou déclaré coupable; g) de bénéficier de la peine la moins sévère, lorsque la peine qui sanctionne l'infraction dont il est déclaré coupable est modifiée entre le moment de la perpétration de l'infraction et celui de la sentence. 
12. Chacun a droit à la protection contre tous traitements ou Punition peines cruels et inusités.

13. Chacun a droit, s'il est contraint de témoigner, à ce qu'aucun témoignage incriminant qu'il donne ne soit utilisé pour l'incriminer dans d'autres procédures, sauf lors de poursuites pour parjure ou pour témoignages contradictoires.

14. La partie ou le témoin qui, lors de procédures, ne com- Interpète prennent pas ou ne parlent pas la langue employée ont droit à l'assistance d'un interprète.

\section{Droits à la non-discrimination}

15. (1) Tous sont egaux devant la loi et ont droit à la même protection de la loi, indépendamment de toute distinction fondée sur la race, l'origine nationale ou ethnique, la couleur, la religion, Egalité devant la loi et protection égale l'âge ou le sexe.

(2) Le présent article n'a pas pour effet d'interdire les lois, programmes ou activités destinés à améliorer la situation des personnes et des groupes défavorisés.

\section{Langues officielles du Canada}

16. (1) Le français et l'anglais sont les langues officielles du Canada; elles ont un statut et des droits et privilèges égaux quant à leur usage dans les institutions du Parlement et du gouvernement du Canada.

(2) La présente charte ne limite pas le pouvoir du Parlement Portée et des législatures d'améliorer le statut du français et de l'anglais ou de l'une de ces langues, ou d'en développer l'usage.

17. Chacun a le droit d'employer la langue officielle de son choix dans les débats et travaux du Parlement.

18. Les lois, les archives, les comptes rendus et les procèsverbaux du Parlement sont imprimés et publiés en français et en anglais, les deux versions des lois ayant également force de loi et celles des autres documents ayant même valeur.

19. Chacun a le droit d'employer la langue officielle de son choix dans toutes les affaires dont sont saisis les tribunaux établis par le Parlement et dans tous les actes de procédure qui en découlent.

20. Chacun a, au Canada, à titre privé, droit à l'emploi de la langue officielle de son choix pour communiquer avec le siège ou l'administration centrale des institutions du Parlement ou du gouvernement du Canada ou pour en recevoir les services; il a le

Langues officielles du Canada

Programmes d'action sociale même droit à l'égard de tout autre bureau de ces institutions situé dans une région du Canada où il est reconnu, conformément aux modalités prévues ou autorisées par le Parlement, q'une partie importante de la population emploie la langue qu'il a choisie.

Travaux du Parlement

Documents parlementaires

Procédures devant les tribunatux établis par le Parlement

Communications entre les administrés et les institutions fédérales 
Maintien en vigueur de certaines dispositions

Droits preservés d'instruction

Continuité d'emploi de la langue d'instruction

Droits et libertés non expressément visés

Primauté de la charte

Droit sur la preuve

Application aux territoires
21. Les articles 16 à 20 n'ont pas pour effet, en ce qui a trait à la langue française ou anglaise ou à ces deux langues, de porter atteinte aux droits, privilèges ou obligations qui existent ou sont maintenus aux termes d'une autre disposition de la Constitution du Canada.

22. Les articles 16 à 20 n'ont pas pour effet de porter atteinte aux droits et privilèges, antérieurs ou postérieurs à l'entrée en vigueur de la présente charte et découlant de la loi ou de la coutume, des langues autres que le française ou l'anglais.

\section{Droits à l'instruction dans la langue de la minorité}

23. (1) Les citoyens canadiens dont la première langue apprise et encore comprise est celle de la minorité francophone ou anglophone de leur province de résidence ont le droit de faire instruire leurs enfants, aux niveaux primaire et secondaire, dans la langue de la minorité dans toute région de la province où le nombre des enfants de ces citoyens justifie la mise sur pied, au moyen de fonds publics, d'installations d'enseignement dans cette langue.

(2) Le citoyen canadien qui change de résidence d'une province à une autre a le droit de faire instruire ses enfants, aux niveaux primaire et secondaire, dans la langue, française ou anglaise, dans laquelle l'un de ses enfants recevait son instruction dans la province de son ancienne résidence, dans toute région de sa nouvelle province de résidence où le nombre d'enfants de citoyens jouissant d'un droit reconnu au présent article justifie la mise sur pied, au moyen de fonds publics, d'installations d'enseignement dans cette langue.

\section{Droits et libertés non expressément visés}

24. La présente charte ne nie pas l'existence des droits et libertés qu'elle ne garantit pas expressément et qui existent au Canada, notamment les droits et libertés des peuples autochtones du Canada.

\section{Dispositions générales}

25. La présente charte rend inopérantes les dispositions incompatibles de toute autre règle de droit.

26. A l'exception de l'article 13, les dispositions de la présente charte ne portent pas atteinte aux lois sur l'admissibilité de la preuve en justice, ni aux pouvoirs du Parlement et des législatures de légiférer en cette matière.

27. Dans la présente charte, les dispositions qui visent les provinces, leur législature ou leur assemblée législative visent également le territoire du Yukon, les territoires du Nord-Ouest ou leurs autorités législatives compétentes. 
28. La présente charte n'élargit pas les compétences législatives de quelque organisme ou autorité que ce soit.

Non-élargisse-

ment des

compétences

législatives

\section{Application de la charte}

29. (1) La présente charte s'applique:

a) au Parlement et au gouvernement du Canada, ainsi qu'à tous les domaines relevant du Parlement, y compris ceux qui concernent le territoire du Yukon et les territoires du NordOuest;

b) à la législature et au gouvernement de chaque province, ainsi qu'à tous les domaines relevant de cette législature.

(2) Par dérogation au paragraphe (1), l'article 15 ne s'applique Restriction que trois ans après l'entrée en vigueur, exception faite de la partie $V$, de la présente loi.

Titre

30. Titre de la présente partie: Charte canadienne des droits Titre et libertés.

PARTIE II

PEREQUATION ET INEGALITES REGIONALES

31. (1) Sous réserve des compétences législatives du Parle- Engagements ment et des législatures et de leur droit de les exercer, le Par- relatifs à à lement et les législatures, ainsi que les gouvernements fédéral et chances provinciaux, s'engagent à:

a) promouvoir l'égalité des chances de tous les Canadiens dans

la recherche de leur bien-être;

b) favoriser le développement économique pour réduire l'inégalité des chances;

c) fournir à tous les Canadiens, à un niveau de qualité acceptable, les services publics essentiels.

(2) Le Parlement et le gouvernement du Canada s'engagent Engagement à prendre les dispositions propres à mettre les provinces en mesure d'assurer les services publics essentiels visés à l'alinéa (1) c) sans relatif aux services publics qu'elles aient à imposer un fardeau fiscal excessif.

PARTIE III

\section{CONFERENCES CONSTITUTIONNELLES}

32. Avant l'entrée en vigueur de la partie $\mathrm{V}$, le premier mi- Conférences nistre du Canada convoque au moins une fois par an une conféconstitutionnelles rence constitutionnelle réunissant les premiers ministres provinciaux et lui-même, sauf si la majorité d'entre eux décide de ne pas la tenir une année donnée. 
Modification à l'égard de certaines provinces

Règles

Idem

Restriction du recours à la procédure provisoire

Entrée en vigueur de la partie $\mathrm{V}$

Proposition de remplacement

Possibilité de mise au point
PARTIE IV

\section{PROCÉDURE PROVISOIRE DE MODIFICATION}

ET REGLES DE REMPLACEMENT

33. Avant l'entrée en vigueur de la partie V, la Constitution du Canada peut être modifiée par proclamation du gouverneur général sous le grand sceau du Canada, autorisée par des résolutions du Sénat et de la Chambre des communes et par l'assemblée législative ou le gouvernement de toutes les provinces.

34. Avant l'entrée en vigueur de la partie $V$, les dispositions de la Constitution du Canada applicables à certaines provinces seulement peuvent être modifiées par proclamation du gouverneur général sous le grand sceau du Canada, autorisée par des résolutions du Sénat et de la Chambre des communes et par l'assemblée législative ou le gouvernement de chaque province à laquelle la modification s'applique.

35. (1) L'initiative des procédures de modification visées aux articles 33 et 34 appartient au Sénat, à la Chambre des communes, à l'assemblée législative d'une province ou au gouvernement de celle-ci.

(2) La résolution adoptée ou l'autorisation donnée, dans le cadre de la présente partie, peut être révoquée à tout moment avant la date de la proclamation qu'elle autorise.

36. Les articles 33 et 34 ne s'appliquent pas aux cas de modification constitutionnelle pour lesquels une procédure différente est prévue par une autre disposition de la Constitution du Canada. La procédure visée à l'article 33 s'impose toutefois, pour modifier la Charte canadienne des droits et libertés, ainsi que les dispositions relatives à la modification de la Constitution, y compris le présent article; cette procédure peut également servir à toute codification ou révision générales de la Constitution.

37. La partie $V$ entre en vigueur à la première des dates suivantes:

a) avec ou sans modification, à la date fixée par proclamation prise conformément à la procédure visée à l'article 33;

b) deux ans après l'entrée en vigueur, exception faite de la partie $\mathrm{V}$, de la présente loi.

Il demeure entendu que, si la tenue d'un référendum s'impose conformément au paragraphe 38(3), la partie $V$ entre en vigueur conformément à l'article 39.

38. (1) Les gouvernements ou assemblées législatives d'au moins huit provinces dont la population confondue représente, selon le recensement général le plus récent à l'époque, au moins quatre-vingts pour cent de la population de toutes les provinces peuvent présenter une proposition commune en vue de remplacer la procédure prévue à l'alinéa $41(1) b)$.

(2) Chaque province concernée peut déposer le texte de la proposition visée au paragraphe (1) auprès du directeur général des élections du Canada dans les deux ans suivant l'entrée en vigueur, 
exception faite de la partie $V$, de la présente loi, étant entendu qu'elle peut retirer le texte au cours de cette période.

(3) Dans les cas où, deux ans après l'entrée en vigueur, excep- Référendum tion faite de la partie $V$, de la présente loi, au moins huit provinces remplissant les conditions démographiques visées au paragraphe (1) n'ont pas retiré leur texte, le gouvernement du Canada fait tenir, dans les deux années suivant l'échéance des deux ans, un référendum pour déterminer laquelle des procédures suivantes sera adoptée:

a) celle qui est prévue à l'alinéa $41(1) b$ ) ou l'eventuelle procédure de remplacement proposée par le gouvernament du Canada après dépôt de son texte auprès du directeur général des élections au moins quatre-vingt-dix jour avant la date du référendum;

b) celle qui fait l'objet de la proposition des provinces.

39. Dans les six mois suivant la date du référendum, une proclamation sous le grand sceau du Canada est prise en vue de faire entrer en vigueur la partie $V$, éventuellement modifiée dans la mesure nécessaire pour incorporer la proposition approuvée par la majorité des votants et pour intégrer les autres aménagements justifiés qui en découlent.

40. (1) Sous réserve du paragraphe (2), le Parlement peut légiférer pour réglementer la tenue du référendum visé au paragraphe 38(3).

(2) Tout citoyen canadien a le droit de vote à l'occasion du Droit de vote référendum visé au paragraphe $38(3)$. Ce droit ne peut, sans motif valable, faire l'objet d'aucune distinction ou restriction.

PARTIE V

PROCEDURE DE MODIFICATION DE LA CONSTITUTION DU CANADA

41. (1) La Constitution du Canada peut être modifiée par Procédure proclamation du gouverneur général sous le grand sceau du Cana- $\begin{aligned} & \text { normale de } \\ & \text { modification }\end{aligned}$ da, autorisée:

a) par des résolutions du Sénat et de la Chambre des communes;

b) par des résolutions des assemblées législatives d'une majorité des provinces; cette majorité doit comprendre:

(i) chaque province dont la population, avant la date de cette proclamation, représentait, selon un recensement général antérieur quelconque, au moins vingt-cinq pour cent de la population du Canada,

(ii) au moins deux des provinces de l'Atlantique dont la population confondue représente, selon le recensement général le plus récent à l'époque, au moins cinquante pour cent de la population de l'ensemble de ces provinces,

(iii) au moins deux des provinces de l'Ouest dont la population confondue représente, selon le recensement général 
le plus récent à l'époque, au moins cinquante pour cent de la population de l'ensemble de ces provinces.

Définitions

"provinces de l'Atlantique"

«provinces de l'Ouest»

Modification autorisée par référendum

Autorisation de référendum

Modification à l'egard de certaines provinces

Modification sans résolution du Sénat

Règles

applicables aux procédures de modification

Idem

Réglementation des référendums

Droit de vote
(2) Les définitions qui suivent s'appliquent au présent article. «provinces de l'Atlantique». Les provinces de la Nouvelle-Écosse, du Nouveau-Brunswick, de l'Île-du-Prince-Edouard et de TerreNeuve.

«provinces de l'Ouest». Les provinces du Manitoba, de la Colombie-Britannique, de la Saskatchewan et de l'Alberta.

42. (1) La Constitution du Canada peut être modifiée par proclamation du gouverneur général sous le grand sceau du Canada, autorisée par un référendum tenu dans tout le pays conformément au paragraphe (2) et lors duquel la modification a été approuvée:

a) d'une part, à la majorité des votants;

b) d'autre part, à la majorité des votants de chacune des provinces dont les résolutions de leur assemblées législatives suffiraient, avec les résolutions du Sénat et de la Chambre des communes, à autoriser la proclamation mentionnée au paragraphe 41(1).

(2) L'ordre de tenue d'un référendum mentionné au paragraphe (1) est donné par proclamation du gouverneur général sous le grand sceau du Canada, autorisée par les résolutions du Sénat et de la Chambre des communes.

43. Les dispositions de la Constitution du Canada applicables à certaines provinces seulement peuvent être modifiées par proclamation du gouverneur général sous le grand sceau du Canada, autorisée par des résolutions du Sénat, de la Chambre des communes et de l'assemblée législative de chaque province à laquelle la modification s'applique.

44. La Constitution du Canada peut être modifiée par proclamation, dans le cadre du paragraphe $41(1)$ ou de l'article 43 , sans une résolution du Sénat autorisant la proclamation, lorsque, dans un délai de quatre-vingt-dix jours suivant l'adoption par la Chambre des communes d'une résolution à cet effet, le Sénat n'a pas adopté une telle résolution et si, après l'expiration de ce délai, la Chambre des communes adopte de nouveau la résolution. Dans la computation du délai ne sont pas comptés les jours pendant lesquels le Parlament est prorogé ou dissous.

45. (1) L'initiative des procédures de modification visées au paragraphe $41(1)$ et à l'article 43 appartient au Sénat, à la Chambre des communes ou à l'assemblée législative d'une province.

(2) La résolution adoptée dans le cadre de la présente partie peut être révoquée à tout moment avant la date de la proclamation qu'elle autorise.

46. (1) Le Parlement peut, sous réserve du paragraphe (2), légiférer pour réglementer la tenue du référendum visé à l'article 42 .

(2) Tout citoyen canadien a le droit de vote lors du référendum visé à l'article 42 ; ce droit ne peut, sans motif valable, faire l'objet d'aucune distinction ou restriction. 
47. Les articles 41,42 ou 43 ne s'appliquen pas aux cas de modification constitutionnelle pour lesquels une procédure différen. te est prévue par une autre disposition de la Constitution $\mathrm{du} \mathrm{Ca}$ nada. La procédure visée aux articles 41 ou 42 s'impose toutefois pour modifier les dispositions relatives à la modification de la Constitution, y compris le présent article; la procédure visée à l'article 41 peut également servir à toute codification ou révision générales de la Constitution.

48. Sous réserve de l'article 50, le Parlement a compétence exclusive pour modifier les dispositions de la Constitution du Canada relatives au pouvoir exécutif fédéral, au Sénat et à la Chambre des communes.

49. Sous réserve de l'article 50, la législature de chaque province a compétence exclusive pour modifier la constitution de celle-ci.

50. Toute modification de la Constitution du Canada portant sur les questions suivantes se fait selon la procédure visée aux articles 41 ou 42:

a) les fonctions de la Reine, celles du gouverneur général et celles des lieutenants-gouverneurs;

b) la Cbarte canadienne des droits et libertés;

c) les engagements énoncés, en matière de 40 péréquation et d'inégalités régionales, à l'article 31 :

d) les pouvoirs du Sénat;

e) le nombre de sénateurs représentant chaque province au

Sénat et les conditions de résidence qu'ils doivent remplir;

f) le droit d'une province d'avoir à la Chambre des communes

un nombre de députés au moins égal à celui de ses sénateurs;

g) les principes de la représentation proportionnelle des provinces à la Chambre des communes prévus par la Constitution du Canada.

51. La rubrique 1 de l'article 91 et la rubrique 1 de l'article 92 de la Loi constitutionnelle de 1867 (antérieurement désignée sous le titre: Acte de l'Amérique du Nord Britannique, 1867), l'Acte de l'Amérique du Nord Britannique ( $n^{\circ} 2$ ), 1949, mentionné au $\mathrm{n}^{\circ} 21$ de l'annexe I de la présente loi, et les parties III et IV de la présente loi sont abrogés.

PARTIE VI

\section{DISPOSITIONS GENERALES}

52. (1) La Constitution du Canada comprend:

a) la Loi sur le Canada;

b) les textes législatifs et les décrets figurant à l'annexe I;

c) les modifications aux textes législatifs et aux décrets mentionnés aux alinéas $a$ ) ou $b$ ).

(2) La Constitution du Canada ne peut être modifiée que con- Modification formément aux pouvoirs conférés par elle.

Restriction du recours à la procédure

normale

Modification

par le Parlement

Modification par les législatures provinciales

Procédure normale de modification

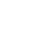

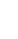

Modifications corrélatives

Constitution du Canada 
Abrogation et nouveaux titres

Modifications corrélatives

Version française de la Constitution du Canada

Versions française et anglaise

Versions française et anglaise Entrée en vigueur

Exception à l'égard des procédures de modification Titres
53. (1) Les textes législatifs énumérés à la colonne I de l'annexe I sont abrogés ou modifiés dans la mesure indiquée à la colonne II. Sauf abrogation, ils restent en vigueur en tant que lois du Canada sous les titres mentionnés à la colonne III.

(2) Toute loi, sauf la Loi sur le Canada, qui fait mention d'une loi figurant à l'annexe I par le titre indiqué à la colonne I est modifiée par substitution à ce titre du titre correspondant mentionné à la colonne III; tout Acte de l'Amérique du Nord Britannique non mentionné à l'annexe $I$ peut être cité sous le titre de Loi constitutionnelle suivi de l'indication de l'année de son adoption et éventuellement de son numéro.

54. Le ministre de la Justice du Canada est chargé de rédiger, dans les meilleurs délais, la version française des parties de la Constitution du Canada qui figurent à l'annexe I; toute partie suffisamment importante est, dès qu'elle est prête, déposée pour adoption par proclamation du gouverneur général sous le grand sceau du Canada, conformément à la procédure applicable à l'époque à la modification des dispositions constitutionnelles qu'elle contient.

55. Les versions française et anglaise des parties de la Constitution du Canada adoptées dans ces deux langues ont également force de loi. En outre, ont également force de loi, dès l'adoption, dans le cadre de l'article 54, d'une partie de la version française de la Constitution, cette partie et la version anglaise correspondante.

56. Les versions française et anglaise de la présente loi ont également force de loi.

57. Sous réserve de l'article 58, la présente loi entre en vigueur au jour fixé par proclamation du gouverneur général sous le grand sceau du Canada.

58. La partie $\mathrm{V}$ entre en vigueur dans les conditions prévues à la partie IV.

59. Titre de la présente annexe: Loi constitutionnelle de 1980; titre commun des lois constitutionnelles de 1867 à 1975 $\left(\mathrm{n}^{\circ}\right.$ 2) et de la présente loi: Lois constitutionnelles de 1867 à 1980. 
ANNEXE I

LOI CONSTITUTIONNELLE DE 1980

ACTUALISATION DE LA CONSTITUTION

Colonne I

Loi visée

1. Acte de 1'Amérique du Nord britannique, 1867, 30-31 Vict., c. 3 (R.-U)

2. Acte pour amender et continuer l'acte trentedeux et trete-trois Victoria, chapitre trois, et pour établir et constituer le gouvernement de la province de Manitoba, 1870, 33 Vict., c. 3 (Canada)

3. Arrêté en conseil de Sa Majesté admettant la Colombie - Britannique, en date du 16 mai 1871

4. Acte de l'Amérique du Nord britannique, 1871 , 34-35 Vict., c. 28 (R.-U.)

5. Arrêté en conseil de Sa Majesté admettant l'Iledu-Prince - Edouard, en date du 26 juin 1873

6. Acte du Parlement du Canada, 1875, 38-39 Vict., c. 38 (R.-U.)

7. Arrêté en conseil de Sa Majesté admettant dans 1'Union tous les territoires et possessions britanniques dans l'Amérique du Nord, et les îles adjacentes à ces territoires et possessions, en date du 31 juillet 1880
Colonne II

Modification

(1) L'article 1 est abrogé Loi constitutionnelle et remplacé par ce qui suit: de 1867

«1. Titre abrégé: Loi constitutionnelle de 1867.»

(2) L'article 20 est abrogé.

(1) Le titre complet est abrogé et remplacé par ce qui suit:

«Loi de 1870 sur le $\mathrm{Ma}$ nitoba.»

(2) L'article 20 est abrogé.

Loi de 1870 sur le Manitoba

Conditions de l'adhésion de la Colombie - Britannique

L'article 1 est abrogé et remplacé par ce qui suit:

«1. Titre abrégé: Loi constitutionnelle de 1871.»

Conditions de l'adhésion de l'Ille-duPrince-Édouard

Loi de 1875 sur le Parlement du Canada

Décret $d u$ conseil sur les territoires adjacents 
Colonne I

Loi visée

8. Acte de l'Amérique du Nord britannique, 1886, 49-50 Vict., c. 35 (R.-U.)

9. Acte du Canada (limites d'Ontario) 1889, 52-53 Vict., c. 28 (R.-U.)

10. Acte concernant l'Orateur canadien (nomination d'un suppléant) 1895 , $2^{\circ}$ session, 59 Vict., c. 3 (R.-U.)

11. Acte de l'Alberta, 1905 , 4-5 Ed. VII, c. 3 (Canada)

12. Acte de la Saskatchewan, 1905, 4-5 Ed. VII, c. 42 (Canada)

13. Acte de l'Amérique du Nord britannique, 1907 , 7 Ed. VII, c. 11 (R.-U.)

14. Acte de l'Amérique du Nord britannique, 1915, 5-6 Geo. V, c. 45 (R.-U.)

15. Acte de l'Amérique du Nord britannique, 1930 , 20-21 Geo. V, c. 26 (R.-U.)

16. Statut de Westminster, 1931, 22 Geo. V, c. 4 (R.-U.)
Colonne II

Modification

Colonne III

Nouveau titre

L'article 3 est abrogé et Loi constitutionnelle remplacé par ce qui suit:

«3. Titre abrégé: Loi

constitutionnelle de 1886.»

Loi de 1889 sur le Canada (frontières de l'Ontario)

La loi est abrogée.

Loi sur l'Alberta

Loi sur la Saskatchewan

L'article 2 est abrogé et remplacé par ce qui suit:

«2. Titre abrégé: Loi constitutionnelle de 1907.»

L'article 3 est abrogé et remplacé par ce qui suit:

«3. Titre abrégé: Loi constitutionnelle de 1915.»

L'article 3 est abrogé et remplacé par ce qui suit: «3. Titre abrégé: Loi constitutionnelle de 1930.»

Dans la mesure où ils s'appliquet au Canada:

a) l'expression «et TerreNeuve» à l'article 1 et au paragraphe $10(3)$ est abrogée;

b) l'article 4 est abrogé;

c) le paragraphe $7(1)$ est abrogé.
Loi constitutionnelle de 1907

Loi constitutionnelle de 1915

Loi constitutionnelle de 1930

Statut de Westminster de 1931 


\begin{tabular}{ccc}
\hline $\begin{array}{c}\text { Colonne I } \\
\text { Loi visée }\end{array}$ & $\begin{array}{c}\text { Colonne II } \\
\text { Modification }\end{array}$ & $\begin{array}{c}\text { Colonne III } \\
\text { Nouveau titre }\end{array}$ \\
\hline
\end{tabular}

17. Acte de l'Amérique du Nord britannique, 1940, 3.4 Geo. VI, c. 36 (R.-U.)

18. Acte de l'Amérique du Nord britannique, 1943, 6.7 Geo. VI, c. 30 (R.-U.)

19. Acte de l'Amérique du Nord britannique, 1946, 9-10 Geo. VI, c. 63 (R.-U.)

20. Acte de l'Amérique du Nord britannique, 1949 , 12-13 Geo. VI, c. 22 (R.-U.)

21. Acte de l'Amérique du Nord britannique ( $\mathrm{N}^{\circ} 2$ ), 1949,13 Geo. VI, c. 81 (R.-U.)

22. Acte de l'Amérique du Nord britannique, 1951, 14-15 Geo. VI, c. 32 (R.-U.)

23. Acte de l'Amérique du Nord britannique, 1952, 1 Eliz. II, c. 15 (Canada)

24. Acte de l'Amérique du Nord britannique, 1960, 9 Eliz. II, c. 2 (R.-U.)

25. Acte de l'Amérique du Nord britannique, 1964, 12-13 Eliz. II, c. 73 (R.-U.)

26. Acte de l'Amérique du Nord britannique, 1965 , 14 Eliz. II, c. 4, Partie I (Canada)
L'article 2 est abrogé et Loi constitutionnelle remplacé par ce qui suit:

«2. Titre abrégé: Loi constitutionnelle de 1940.»

La loi est abrogée.

La loi est abrogée.
L'article 3 est abrogé et remplacé par ce qui suit:

«3. Titre abrégé: Loi

sur Terre-Neuve.»

La loi est abrogée lors de l'entrée en vigueur de l'article 51 de la Loi constitutionnelle de 1980.

La loi est abrogée.

La loi est abrogée.

L'article 2 est abrogé et remplacé par ce qui suit:

«2. Titre abrégé: Loi constitutionnelle de 1960.»

L'article 2 est abrogé et remplacé par ce qui suit:

«2. Titre abrégé: Loi constitutionnelle de 1964.»

L'article 2 est abrogé et Loi constitutionnelle remplacé par ce qui suit: «2. Titre abrégé de la présente partie: Loi constituionnelle de 1965.»
Loi sur Terre-Neuve

Loi constitutionnelle de 1960

Loi constitutionnelle de 1964 de 1965 
Colonne I

Loi visée

27. Acte de l'Amérique du Nord britannique, 1974, 23 Eliz. II, c. 13, Partie I (Canada)
Colonne II Modification

L'article 3, modifié par le Loi constitutionnelle paragraphe 38(1) de la loi de 1974

25-26 Elizabeth II, c. 38

(Canada), est abrogé et remplacé par ce qui suit:

«3. Titre abrégé de la présente partie: Loi constitutionnelle de 1974.»

28. Acte de l'Amérique du Nord britannique, 1975, 23-24 Eliz. II, c. 28, Partie I (Canada) Nouveau titre

Loi constitutionnelle $\mathrm{n}^{\circ} 1$ de 1975
Colonne III
L'article 3, modifié par l'article 31 de la loi 25-26 Elizabeth II, c. 28 (Canada), est abrogé et remplacé par ce qui suit:

«3. Titre abrégé de la présente partie: Loi constitutionnelle $n^{\circ} 1$ de 1975.»

29. Acte de l'Amérique du Nord britannique $\mathrm{n}^{\circ} 2$, 1975, 23-24 Eliz. II, c. 53 (Canada)

L'article 3 est abrogé et Loi constitutionnelle remplacé par ce qui suit: «3. Titre abrégé: Loi constitutionnelle $n^{\circ} 2$ de $\mathrm{n}^{\circ} 2$ de 1975 1975.» 